\title{
Dietary Protein and Carbohydrates Affect Immune Function and Performance in a Specialist Herbivore Insect (Manduca sexta) ${ }^{*}$
}

\author{
J. Keaton Wilson ${ }^{\dagger}$ \\ L. Ruiz \\ G. Davidowitz \\ Department of Entomology, University of Arizona, Forbes \\ 410, PO Box 210036, Tucson, Arizona 85721
}

Accepted 10/11/2018; Electronically Published 11/29/2018

Dryad data: http://dx.doi.org/10.5061/dryad.d7b5p01b.

\begin{abstract}
Nutrition structures ecology and evolution across all scales of biological organization. It is well known that nutrition can have direct effects on performance and fitness, but indirect effects on physiological systems that mediate biotic interactions have been studied less frequently. Here, we focus on the interaction between nutrition, performance, and the immune system in a specialist herbivorous insect, Manduca sexta. We used a conceptual framework in nutritional ecology (the geometric framework) to examine how changes in diet quality affect aspects of the immune system used for defense against parasitoids. We raised caterpillars throughout their entire larval development on five different experimental diets that varied in protein and carbohydrate content and measured five aspects of the immune system: encapsulation, phenoloxidase activity, prophenoloxidase activity, total hemolymph protein, and hemocyte density. Overall, different parts of the immune function varied in response to interactions between carbohydrates, protein, and intake, but protein reductions had the largest impactsmostly detrimental. In addition, our data suggest that diet quality mediates the relationship between performance (growth and survival) and immune function, as well as trade-offs among different components of immune function. Our work is the first to examine the interplay between nutrition, performance, and immune function with the geometric framework in a specialist insect herbivore.
\end{abstract}

*This paper was submitted in response to a call for papers for a Focused Issue on "Ecoimmunology."

†Corresponding author; email: keatonwilson@email.arizona.edu.

Physiological and Biochemical Zoology 92(1):58-70. 2019. (C) 2018 by The University of Chicago. All rights reserved. 1522-2152/2019/9201-8032\$15.00. DOI: $10.1086 / 701196$
Keywords: carbohydrates, geometric framework, immune function, Manduca, protein.

\section{Introduction}

Nutrition plays a critical role in structuring ecology and evolution across all scales of biological organization, from the evolution of cellular and physiological systems of energy conversion to patterns of species distribution. Nutritional ecology integrates information about organismal-level processes to address how phenotypes function in the field and how they affect the surrounding environment (Raubenheimer et al. 2009). Animals in the field experience variation in nutritional quality, which has cascading effects on different physiological systems and can change how organisms interact with their environment as well as directly modify performance and fitness (e.g., Mayntz and Toft 2001; Awmack and Leather 2002; Couture et al. 2010). Insect herbivores have provided a particularly fruitful source of study for biologists interested in how nutrition can affect ecology. This interest is in part because the nutritional space generated by hosts plants is complex, presumably under natural selection, and includes adaptive chemicals that act as deterrents or toxins to herbivores (Rosenthal and Berenbaum 2012). Interest has also stemmed from the idea that multitrophic interactions are important in structuring these systems and are often mediated by the plants being attacked (Dicke 2000). The effects of diet quality on insect performance and fitness have been examined frequently with emphasis on plant secondary chemistry, which is often useful in pest-management applications (Rattan 2010). Simultaneously, work on the insect immune system has demonstrated it to be complex and multipronged, capable of protecting an insect against a variety of pathogens, parasites, and parasitoids (Schmid-Hempel 2003, 2005; González-Santoyo and CórdobaAguilar 2012). Though often studied separately, these two pieces of insect biology have been infrequently examined in concert. Here, we explore the role that nutrition plays in modifying the effectiveness of the insect immune system and how these patterns compare to the effects of nutrition on measures of performance and fitness.

Researchers frequently use the growth rate or body size of insects as measures of fitness when assessing the quality or suitability of different host plants (Mayntz and Toft 2001; Stiling and Cornelissen 2007; Diamond et al. 2010). Growth and body size make good proxies for fitness because they are relatively easy to measure and are often strongly correlated with fitness (Arnold 1983). However, fitness is a complex, multi- 
faceted trait, and other factors besides size and growth (such as immune function) often play a large determining role. Certainly, nutrition can affect performance and fitness directly, but it can also modify behavior and affect the function of physiological systems that contribute to survival or fecundity and thus fitness, indirectly (Karl et al. 2010). For example, a given host plant might convey benefits to a female moth in terms of growth but adversely affect her immune system, leaving her open to attack by parasitoids and pathogens. To fully understand the effect that nutrition has on the ecology and evolution of an organism, we need a more complete understanding of the indirect roles it may play in shaping fitness, particularly through important physiological systems such as the immune system.

Animal immune systems are multipurpose and serve to defend against a number of threats, including bacteria, viruses, fungi, and parasites (Calder 2013). Though there are mechanistic similarities between insect immune systems and the immune systems of other animals, there are also substantial differences. The insect immune system can be broken into general functional categories, including physical barriers such as the cuticle, invader-recognition systems, and targeted and untargeted molecular responses (Schmid-Hempel 2003). The encapsulation response of insects has been well studied and serves as the main line of defense against attacking parasitoids. Encapsulation is generated by the prophenoloxidase (proPO) cascade to deposit melanin around invaders, eventually completely engulfing them (Schmid-Hempel 2003). Here, we focus mainly on components of the immune system (i.e., the proPO cascade and encapsulation response) that defend insects against parasitoids because (1) parasitoids have been shown to have strong impacts on herbivore performance and fitness (Wilson and Woods 2015, 2016) and strong population-level effects (Bonsall and Hassell 1997; Lin and Ives 2003) and (2) wild populations of our study organism (Manduca sexta; Lepidoptera: Sphingidae) are frequently attacked by a variety of parasitoid species in the field (Mira and Bernays 2002; Wilson and Woods 2015,2016) Though some subsystems of immunity overlap in function (e.g., phenoloxidase [PO] production has negative impacts on bacterial pathogens but also leads to encapsulation, which typically deals with larger invading organisms), many derive from different biochemical pathways and can be considered separate arms of the same physiological system. As such, they may trade off with each other, so that a strong immune response to one threat may hamper the ability to fight off another (Cotter et al. 2004; Rantala and Roff 2005). These trade-offs may be further complicated by changes in the nutritional content of a given diet.

The geometric framework (GF) provides a powerful tool in assessing how changes in diet quality can affect the performance of individual physiological systems and ultimately wholeorganism performance and fitness (Raubenheimer and Simpson 1993, 1999; Raubenheimer et al. 2009). The central premise of the GF is that a given source of nutrition can be represented as a multidimensional hypervolume, with each axis representing a different nutritional component. Carbohydrates, protein, and lipids are the components typically plotted, but the GF can be extended to all components of a diet, including defensive secondary compounds present in plant tissue (Simpson and Raubenheimer 2001; Deans et al. 2016). The GF has been used to study a variety of topics in the field of nutritional ecology, including behavior (Raubenheimer and Simpson 1993), optimal nutrient foraging strategies (Simpson et al. 2004), determining how complex interactions between diet components can shape the effectiveness of different physiological systems (Ponton et al. 2011), and understanding how nutrition can affect life-history trade-offs (Rapkin et al. 2018). Here, we use it to assess the performance of specialist, herbivorous insects in a two-dimensional nutritional space (carbohydrates and protein) and different components of the insect immune system.

Nutritional ecologists have been interested in understanding the effects of diet quality on immunity for some time and work in other frameworks (e.g., ecological stoichiometry; Sterner and Elser 2002) has shown strong effects. Historically, nutritional ecology and interactions with immunity have been examined through the lens of quantitative resource constraint, where organisms attempt to maximize intake of a single nutritional resource (Cotter et al. 2011). However, use of the GF has led others to develop the qualitative resource hypothesis (Cotter et al. 2011), which posits that nutritional blends, rather than individual resources, are the intake targets that are important in driving physiological function. If nutritional blends are more important in shaping patterns of performance and immune function than individual components of nutrition, or energy limitation, then the expectation is that factors should vary in nutrient space in complex ways, as outlined by Cotter and colleagues (2011). Here, we test the qualitative resource hypothesis by examining the effects of carbohydrate and protein nutritional space on the growth performance and immune function in a specialist herbivorous insect.

We use the tobacco hornworm (M. sexta) in a series of feeding experiments that use artificial diets to explore how changes in nutrient space can affect performance and immune function (both constitutive and challenged). Our work addresses three main questions: (1) How does performance (growth and survivorship) of caterpillars vary with changes in the protein and carbohydrate content of diets? (2) How do different components of the immune system vary with dietary changes? (3) How do performance and immune function trade off when interacting with varying nutritional content? Though there has been some work examining the interaction between immune function and nutrition in the context of the GF (Cotter et al. 2011), the research outlined here provides novel insights by incorporating the effects of dietary nutrient manipulations during the entire course of larval development and examines trade-offs between immune function and performance under different nutrition regimes. Furthermore, to our knowledge, this work is the first to examine the interplay between nutrition, performance, and immune function with the GF in a specialist insect herbivore. By better understanding the role that nutrition plays in shaping the interaction between immune function and 
growth, we gain insight into the more complex role it has in shaping the ecology and evolution of specialist herbivorous insects, the plants they live on, and the third trophic level, parasitoids that attack them (Kaplan et al. 2016).

\section{Methods and Material}

\section{Larval Care and Diet Formulations}

Manduca sexta were from a colony at the University of Arizona and were raised at $27^{\circ} \mathrm{C}$ in growth chambers under a $16 \mathrm{~L}: 8 \mathrm{D}$ photocycle. Eggs were collected from a large mating chamber containing approximately 50 pairs of moths, and hatchling caterpillars were selected pseudorandomly and placed on artificial-diet treatments within $2 \mathrm{~h}$ of emergence. We raised caterpillars on a standard artificial wheat germ-based diet (Davidowitz et al. 2003) as a control or on one of four experimental diets: low protein, medium protein, low carbohydrate, and medium carbohydrate (table 1). Experimental diet formulations and calculations of ratios of protein to carbohydrates (P:C; table 1) came from calculations used in Ojeda-Avila et al. (2003). Reductions in protein or carbohydrates compared to the control diet were achieved by replacing casein and sucrose with nonnutritive cellulose (Alphacel, ICN Biomedicals, Aurora, OH), a technique used to generate diets of different nutritional qualities (Davidowitz et al. 2003; Ojeda-Avila et al. 2003). Our diet formulations were designed to test differences in macronutrient ratio, but they also varied to a small degree in total macronutrient density (table 1); we address potential effects of these differences in "Discussion." In addition, though other researchers report intake targets for $M$. sexta of 1:1 P:C (Thompson and Redak 2005), we chose to use the $\mathrm{P}: \mathrm{C}$ ratio of the diet that our colony typically consumes $(31: 37$, a relatively small difference from $1: 1)$ for the control diet, for continuity with the practices used in our colony population of $M$. sexta.

\section{Weight Measurements and Food Intake}

Manduca sexta eggs were placed in growth chambers in $32 \times$ $23 \times 5-\mathrm{cm}$ trays with a section of raised wire mesh and blocks of control diet. Caterpillars were allowed to hatch onto control diet. However, shortly after hatching (within approx. $6 \mathrm{~h}$ ), they were removed from the tray, weighed (Mettler Toledo XS3DU, Columbus, $\mathrm{OH})$, and placed randomly into 1-ounce $(29.57-\mathrm{mL})$ plastic cups (Dart Container, Mason, MI) with a perforated lid, along with a piece of preweighed diet. Caterpillars and diet were weighed throughout development, at intervals of between 24 and $48 \mathrm{~h}$, which varied depending on life stage and when caterpillars were tested for immune function at the end of development. During the fourth instar, caterpillars were moved to larger, 9-ounce (266.16-mL) plastic cups (Dart Container), old food was removed, and new food was added. Throughout the experiment, food was added or removed as needed (checked and weighed at intervals of $24-48 \mathrm{~h}$ ) so that caterpillars had adequate supplies of fresh diet throughout development. In eight different rounds of trials, 258 caterpillars were measured. Of these, 114 caterpillars died at some point during development and were not included in analysis (mortality was high in some experimental diet treatments; see table 1). In addition, data from one round of experiments were also not included in analysis, because of a temporary wave of infection in our colony leading to decreased growth and increased mortality in individuals on the control diet in that round. In total, data from 140 caterpillars that survived until the end of the fifth (last) instar were used in subsequent analyses.

\section{Measures of Immune System Function}

Caterpillars at the end of the fifth instar (between 6.5 and $11.5 \mathrm{~g}$ ) but before the onset of wandering behavior (Dominick and Truman 1984) were removed from cups and weighed one final time before being subjected to measures of immune system function. The onset of wandering is initiated by a reduction in juvenile hormone, which is dependent on a threshold critical weight of $7 \mathrm{~g}$ (Davidowitz et al. 2003, 2016). We measured immune function of caterpillars at the end of the fifth instar for two reasons: (1) this developmental stage is frequently attacked by Drino rhoeo (Tachinidae), a parasitoid fly, and other parasitoids (Mira and Bernays 2002; Wilson and Woods 2015), and (2) there can be large changes in immune function after the onset of wandering (Hung and Boucias 1996; Miranda et al. 2013).

After weighing, caterpillars were kept in 9-ounce plastic cups at room temperature until they were ready to be tested for immune function. Caterpillars were placed, one at a time, in a small Styrofoam cooler filled with crushed ice for approximately 2 min until movement was noticeably slowed. The caterpillar was removed from the ice and placed on the lab

Table 1: Formulations of experimental diets and caterpillar survival

\begin{tabular}{lcccccc}
\hline Diet & $\begin{array}{c}\text { Protein } \\
(\mathrm{g} / \mathrm{L})\end{array}$ & $\begin{array}{c}\text { Carbohydrates } \\
(\mathrm{g} / \mathrm{L})\end{array}$ & $\mathrm{P}: \mathrm{C}$ & $\begin{array}{c}\text { Macronutrient content } \\
\text { compared to control }(\%)\end{array}$ & $\begin{array}{c}\text { Survival } \\
(\%)\end{array}$ & $\begin{array}{c}\text { Reduction from } \\
\text { control }(\%)^{\mathrm{a}}\end{array}$ \\
\hline Control & 86.06 & 101.751 & $.84: 1$ & 100 & 71.2 & $\ldots$ \\
Low protein & 53.06 & 101.751 & $.51: 1$ & 82 & 14.2 & 38.3 \\
Medium protein & 62.06 & 101.751 & $.6: 1$ & 85 & 55.0 & 27.9 \\
Low carbohydrate & 86.06 & 73.751 & $1.2: 1$ & 88 & 70.0 & 27.5 \\
Medium carbohydrate & 86.06 & 80.251 & $1.07: 1$ & 69.2 & 21.1 \\
\hline
\end{tabular}

Note. $\mathrm{P}: \mathrm{C}=$ protein: carbohydrate ratio.

${ }^{a}$ Reduction in protein or carbohydrate. 
bench. A steel insect pin sterilized in $70 \%$ ethanol (size 00 , Bioquip, Rancho Dominguez, CA) was used to make a small hole below the spiracle on the hindmost midbody segment with a proleg (segment A6), on the left side of the caterpillar. Hemolymph that leaked from the incision was collected and added to an Eppendorf tube kept on ice. A small piece (approx. $3 \mathrm{~mm}$ in length) of preweighed fishing line (10-lb. test, $0.30-\mathrm{mm}$ diameter; Pure Fishing, Columbia, SC) was inserted into the incision, angled toward the leg (not perpendicular), and pushed completely into the body of the animal. The fishing line is designed to mimic an invading parasite - the insect immune system recognizes it as foreign and encapsulates it. If enough hemolymph was collected (a minimum of $\sim 80 \mu \mathrm{L}$ ), the animal was placed back in a 9-ounce plastic cup with diet and left in the environmental growth chamber for $24 \mathrm{~h}$. Afterward, the caterpillar was frozen in a $-20^{\circ} \mathrm{C}$ freezer until implant removal. If not enough hemolymph was collected, the steel pin was resterilized and used to make a small dorsal incision; additional hemolymph was collected and added to the hemolymph collected from the implant incision. Eppendorf tubes of hemolymph were kept on ice until they were prepped for storage.

Hemolymph was split into two Eppendorf tubes for storage. In one tube, $10 \mu \mathrm{L}$ of a $1: 1$ mixture of EDTA (ethylenediaminetetraacetic acid) buffer and glycerol was mixed with $10 \mu \mathrm{L}$ of hemolymph. These samples were used for hemocyte counts. The rest of the hemolymph was stored undiluted for $\mathrm{PO}$ and protein assays. Samples were frozen in a $-20^{\circ} \mathrm{C}$ freezer until processing.

\section{Hemolymph PO, ProPO, and Protein}

Hemolymph $\mathrm{PO}$ and proPO were determined spectrophotometrically, with dopamine as the substrate (Cotter et al. 2008). Eppendorf tubes of hemolymph were removed from the freezer and placed in a Styrofoam cooler full of crushed ice. Eight microliters of each sample was diluted with $360 \mu \mathrm{L}$ of $10 \mathrm{mM}$ $\mathrm{NaCac}$ (sodium cacodylate) buffer and then split into two Eppendorf tubes. We added $20 \mu \mathrm{L}$ of $\mathrm{NaCac}$ to one tube used for PO analysis. To the other tube, we added $20 \mu \mathrm{L}$ of a solution of $20 \mathrm{mg} / \mathrm{mL}$ chymotrypsin in NaCac buffer, used for proPO analysis. Samples were incubated at between $23^{\circ}$ and $25^{\circ} \mathrm{C}$ for $1 \mathrm{~h}$. Ninety microliters of each sample was added to a 96-well microtiter plate (PO on one half of the plate and proPO on the other half), in duplicate. Ninety microliters of a $4 \mathrm{mM}$ dopamine solution was then added to each well, and the plate was analyzed at $25^{\circ} \mathrm{C}$ (ThermoFisher Scientific Multiskan Go spectrophotometer, Thermo Scientific, Waltham, MA). Absorbance readings were taken at $492 \mathrm{~nm}$ every $12 \mathrm{~s}$ for $10 \mathrm{~min}$. Activity was expressed as the maximum slope of the line over the time measured, which is the linear phase of the reaction (Cotter et al. 2011).

Protein was measured with the BioRad protein assay kit (BioRad, Hercules, CA), with bovine serum albumin (BSA) as the standard. Five microliters of diluted hemolymph solution was added to each well of a 96-well microtiter plate. Two hundred microliters of filtered dye reagent was added to the wells containing hemolymph, along with a set of BSA standards. Absorption was measured at $25^{\circ} \mathrm{C}$ on a ThermoFisher Scientific Multiskan Go spectrophotometer.

\section{Encapsulation Assay}

Caterpillars were taken out of a $-20^{\circ} \mathrm{C}$ freezer and placed in a $150 \times 15-\mathrm{mm}$ petri dish (Falcon, Becton Dickinson, Franklin Lakes, NJ). A $50-\mathrm{mL}$ beaker was filled with $\sim 5 \mathrm{~mL}$ of $70 \% \mathrm{EtOH}$ (ethyl alcohol) to sterilize the scalpel, forceps, and scissors. Approximately $3 \mathrm{~mL}$ of water was poured into the petri dish to soften the frozen caterpillar. The head and tail were removed with sterilized scissors and placed in the petri dish's lid. Using a scalpel, we made an incision on the ventral side of the caterpillar from the approximate midpoint to the posterior end. If any small piece of fishing line was visible from the split made by the incision, it was removed for weighing; otherwise, skin was pulled back with forceps to expose the fishing line. Extracted fishing line was then place in a prelabeled and weighed $8 \times 20$-mm aluminum micro weighing dish (Wheaton, Millville, NJ) before being placed inside a drying oven at $51^{\circ} \mathrm{C}$ for $48 \mathrm{~h}$. After drying, fishing line and encapsulated tissue were weighed on a microbalance (Mettler Toledo XS3DU) with a resolution of $0.001 \mathrm{mg}$, and the initial line weight was subtracted from this value to obtain dry-tissue weight.

\section{Hemocyte Counts}

Hemolymph samples were removed from the $-20^{\circ} \mathrm{C}$ freezer and put in a Styrofoam holder for approximately $2 \mathrm{~min}$ to thaw. We then used a 200-uL VMR pipette to pipette $90 \mathrm{uL}$ hemolymph into a Bright-Line 0.1-mm-deep hemocytometer (Hausser Scientific, Horsham, PA). We waited approximately $3 \mathrm{~min}$ for capillary action to spread the hemolymph cells across the $5 \times 5$ hemocytometer grid. Then we placed the hemocytometer under the microscope's lens (Leica M205 A; focus: $0.342 \mathrm{~mm}$, magnification: $136.3 \times$ ), and hemolymph cells (hemocytes) were counted one by one in every square in the grid. The hemocyte count was recorded in a $5 \times 5$ table in which each table cell corresponded to the square location in each grid. The total hemocyte count was the sum of hemocyte counts in all table cells. After counting all the hemolymph cells, a picture of the hemocytometer grid was acquired. The hemocytometer and the cover glass were sprayed with water and $70 \% \mathrm{EtOH}$ to sterilize them. A nonabrasive wipe was used to clean the hemocytometer and cover glass. This process was repeated every time a new hemolymph sample was taken.

\section{Data Analysis}

All statistical analyses were done in R (ver. 3.3.2; http://www.R -project.org). Interpolated surfaces of performance and immune function in nutrient space were generated by using the Krig function to generate surfaces in nutrient space (with amount of ingested protein and carbohydrates on the $X$ - and $Y$ axes, respectively) in the fields package in R. Kriging, typically used in geostatistics, interpolates by modeling using a Gaussian process determined by prior covariances (Cressie 1990). Cat- 
erpillar growth rate was modeled with the best-fit linear mixedeffects model (nlme package), with log-transformed weight as the response variable; time, diet treatment, and their interaction as fixed effects; and individual caterpillar identity as a random effect. We used a model-selection approach to determine that a random-slopes-and-intercepts model performed best (randomslopes-and-intercepts Akaike information criterion [AIC]: 1,700, random-intercepts AIC: 1,872) and used subsequent estimates of modeled individual growth rate to generate performance indices for later analyses (table A1). A MANOVA was used to determine the overall effect of diet treatment on immune function, and individual ANOVAs were used to determine the effects of diet treatment on the individual components of immune function. Performance indices were calculated by modeling an individual caterpillar's growth rate and scaling that growth rate by the average survival of the dietary treatment that caterpillar was reared on. This two-part method of calculating performance has been used successfully in other studies of larval performance (Cotter et al. 2011). We used ordinary least squares (OLS) linear regression models to determine whether different components of the measured immune response were correlated with one another, as well as to determine the relationship between immune function, performance, and diet. Though standard major-axis regressions are often used in instances where variables on both axes contain error, it is appropriate to use OLS when the variation in the variable on the $Y$-axis is significantly greater than the variable on the $X$-axis (Legendre and Legendre 2012), as in our case.

\section{Results}

Caterpillar Feeding, Performance, and Survival on Experimental Diets

Manduca sexta larvae had the highest growth rate on the control and carbohydrate-reduced diets (fig. 1). Among these three diets, average growth rates were statistically similar (fig. 1). Caterpillars that consumed protein-deficient diets had slower growth rates than those on the control diet (table A1; low protein: $t_{673}=-13.488, P<0.0001$; medium protein: $t_{673}=$ $-12.918, P<0.0001$ ), with the low-protein diet having the lowest growth rate of all the diet treatments (fig. 1). Survival also varied among diets, with the lowest being $14.2 \%$ on the low-

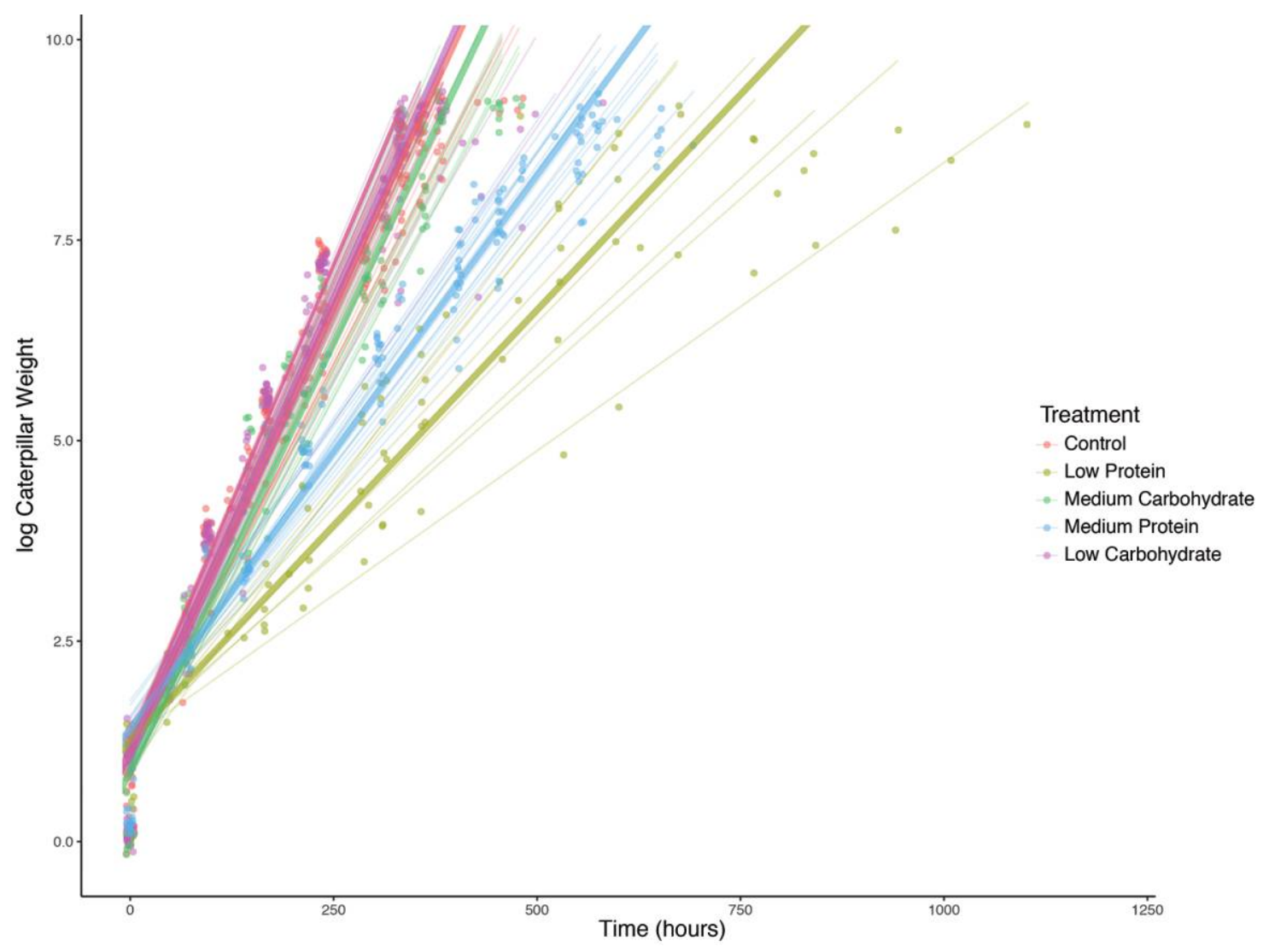

Figure 1. Manduca sexta caterpillar growth over time as a response to experimental diet treatment. Weight data are log transformed, individual measurements of weight colored by diet treatment and are slightly jittered for clarity. Thin lines represent the individual linear growth rates predicted by the random-slopes-and-intercepts linear mixed-effects model, while thick lines represent the overall population model for each treatment group. Note that the control and reduced-carbohydrate diets show significant overlap (they are not significantly different from each other; table A1) and are difficult to distinguish from each other and that the medium- and low-protein diets show significantly reduced growth rates compared to the control (medium protein: $P<0.0001$, low protein: $P<0.0001$ ). 
protein diet, ranging up to $71.2 \%$ on the control diet (table 1). Larvae in low-protein diets appeared to have a difficult time successfully shedding exuvia during molts between instars, which led to constrictions of the body and subsequent death.

Caterpillar performance varied across nutrient space, with the highest values of performance centered on the control diet $(\mathrm{P}: \mathrm{C}$ ratio $=31: 37)$ at moderate intake levels (fig. $2 a)$. However, performance remained high with reductions in carbohydrate content. Protein reduction had the strongest effects on caterpillar performance, with steep drops in performance in both mediumand low-protein treatments across all intake levels (fig. $2 a$ ).

Average caterpillar intake levels were statistically similar among the control, medium-carbohydrate $(t=1.825, P=$ $0.070)$, low-carbohydrate $(t=-1.080, \quad P=0.2821)$, and medium-protein diets $(t=0.0 .654, P=0.514)$ but significantly higher in the low-protein diet $(t=3.003, P=0.003$; fig. 3), though the variance in individual intake levels was much higher in this treatment than in other diets.

\section{Dietary Effects on Immune Function}

Dietary treatment had, overall, a significant effect on immune function, according to MANOVA analysis $\left(F_{4,20,196}=2.3208\right.$, $P=0.002$; table 2$)$, with marginal effects on encapsulation rates $\left(F_{4}=2.2831, P=0.073\right)$ and strong effects on $\mathrm{PO}\left(F_{4}=\right.$ $4.8585, P=0.002)$ and hemolymph protein $\left(F_{4}=5.9004\right.$, $P<0.001)$. ProPO and hemocyte density were unaffected by diet treatment $\left(F_{4}=1.1801, P=0.331\right.$ and $F_{4}=1.5229$, $P=0.2098$, respectively).

Patterns of encapsulation were driven mostly by changes in the protein content of diets, with lower protein levels resulting in lower levels of encapsulation (fig. $2 b$ ). Interestingly, the highest encapsulation levels were at relatively low intake levels and were not centered around the control diet but were found at low carbohydrate levels. In general, caterpillars fed diets with low carbohydrates and higher protein had stronger encapsulation responses. PO levels were strongly affected by the protein content of the diets, as well as the overall intake rates, with the highest PO levels at moderate intakes, centered around the control diet (fig. 2c). ProPO levels were unaffected by changes in $\mathrm{P}: \mathrm{C}$ ratios in statistical analyses $\left(F_{4}=19.9673, P=0.1077\right)$, which is reflected in figure $2 d$. Intake levels had the biggest impact on ProPO, with higher levels of ProPO at low and moderate intake levels. Hemolymph protein levels varied in more complex ways across diet treatments and intake levels (fig. 2e). Hemocyte density decreased with decreases in protein, in a pattern similar to changes in encapsulation and PO (fig. $2 f$ ).

Some measures of immune function were correlated with each other, while others were not (this was, in part, the reason for using a MANOVA for analyzing overall effect of diet treatment on immune function; table 2). Overall, PO levels were positively correlated with proPO levels $\left(F_{1,90}=153.8, R^{2}=\right.$ $0.63, P<0.001$; fig. A1), though the relationship depended on the diet treatment. The low-protein diet produced higher levels of PO, compared to ProPO, than the rest of the diet treatments $(t=2.115, P=0.0340)$, and the medium-protein diet produced lower levels of PO, compared to ProPO $(t=$ $-2.951, P=0.0041)$. There was also a significant positive correlation between PO levels and encapsulation $\left(F_{1,26}=13.35\right.$, $\left.P=0.001, R^{2}=0.31\right)$, but only in the caterpillars fed the control diet - this correlation disappeared on the other experimental diets. Interestingly, there was no significant correlation among other measures of immune function.

\section{Trade-Offs between Immune Function and Performance}

Overall, caterpillars showed no trade-off between performance and the measured components of immune function. Interestingly, three components of immune function (PO, ProPO, and encapsulation) had significant positive correlations with growth rate, while total hemolymph protein showed a significant negative correlation (fig. 4 ; table 3 ). However, this pattern was mostly due to differences among diet treatments - caterpillars on the low- and medium-protein diets had lower average performance indices and measures of immune function, compared to those on other diets (fig. 4). We found that adding the dietary treatment to models did not improve overall model accuracy, and immune function response was modeled across all treatment groups (fig. 4; table 3).

\section{Discussion}

We show that, overall, changes in diet composition have strong influences on both the growth performance and the immune function of Mandica sexta caterpillars. Trade-offs among different components of organismal function have been studied frequently across diverse fields such as development (Davidowitz et al. 2016), life-history theory (Stearns 1992; Martin et al. 2007), allometry (West et al. 1999), and sexual selection (McGraw et al. 2011; McCullough and Emlen 2013) and are important in shaping the way in which selection operates on different (and often competing) components of organismal function that can affect fitness. There is strong evidence for reproductive activity trading off with immunity in female insects (Vijendravarma et al. 2009; Schwenke et al. 2016) as well as for trade-offs between immune system function and performance in a number of organisms (Soler et al. 2003: Ardia 2005; Lochmiller and Deerenberg 2016), including insects (Rantala and Roff 2005; Bascuñán-García et al. 2010). Trade-offs are not a universal phenomenon, however; results are often mixed, differ among taxonomic groups, and may not be symmetric (i.e., selection for growth can have strong negative effects on immune function, but not vice versa; van der Most et al. 2011). Some researchers attribute non-trade-offs to a lack of survival costs and ecological heterogeneity (in the case of sexually selected weapons; McCullough and Emlen 2013), while others hypothesize that the mechanism of non-trade-offs is a disparity in the energy requirements among competing systems (van der Most et al. 2011). The reality is likely a mixture of different processes, given that researchers often examine trade-offs on different scales of biological organization (e.g., sexually selected horns and reproductive systems vs. immune function and/or growth and development) and in organisms that occupy different ecological niches and have vastly 


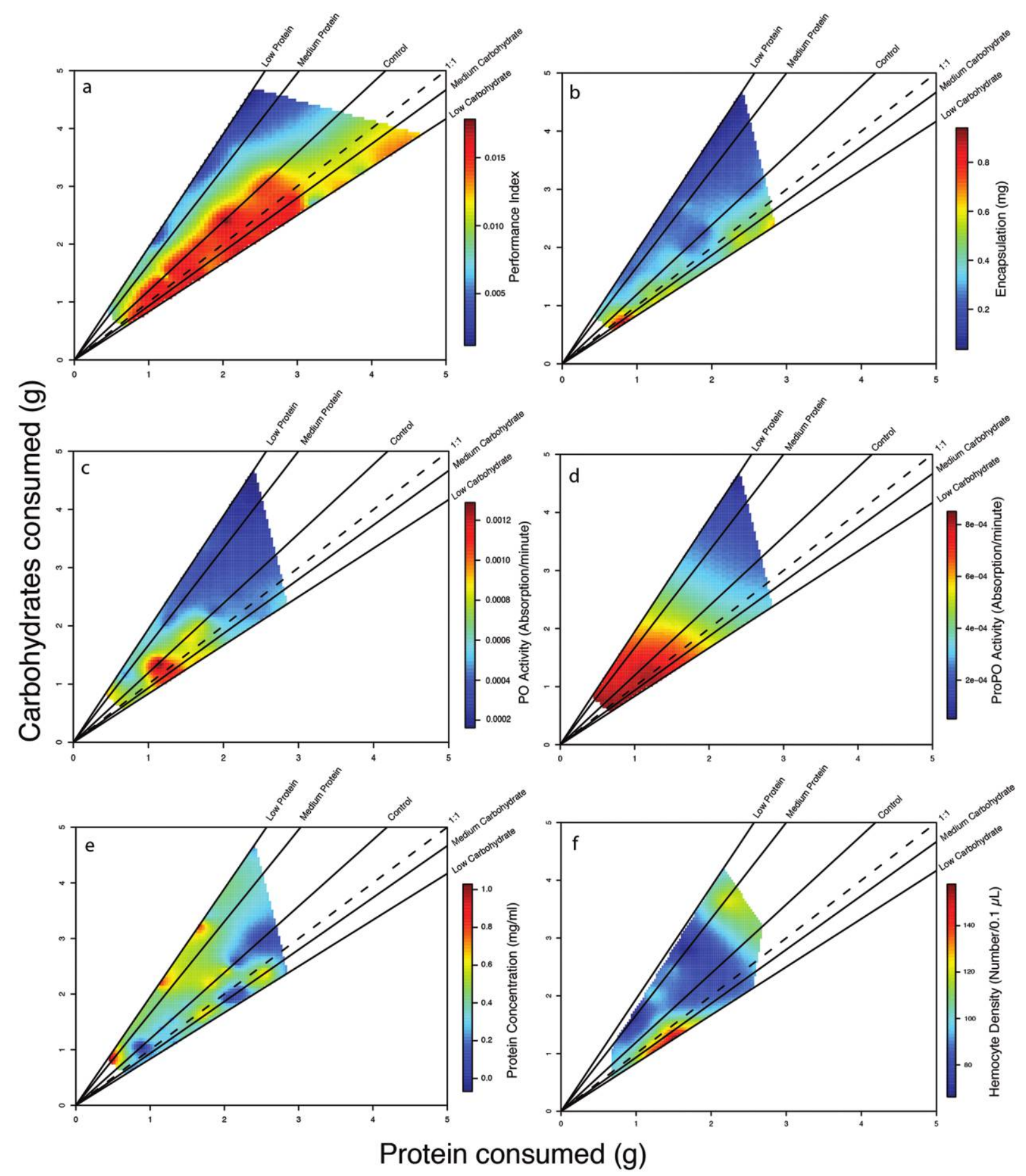

Figure 2. Growth performance and immune function of Manduca sexta larvae in nutrient space. In each panel, hot colors (e.g., red) indicate high values and cool colors (e.g., blue) indicate low values. Each diagonal solid black line represents a nutritional rail provided by each diet, whereas the 1:1 rail is represented by a dashed line. The $X$ - and $Y$-axes of each panel depict the amount of protein and carbohydrates consumed by larvae, respectively. Because individual caterpillars did not have a choice of diets, they cannot move off the rails but can only slide up and down, depending on how much total food they consume. $a$, Performance index of caterpillars on different experimental diets. The performance index is calculated by scaling an individual caterpillar's growth rate by the survival rate in a particular diet treatment. $b$, Encapsulation response of caterpillars plotted in nutrient space. Encapsulation was measured as milligrams of dry weight of encapsulated tissue surrounding nylon implants after $24 \mathrm{~h}$. $c$, Phenoloxidase (PO) response of caterpillars plotted in nutrient space. $d$, Prophenoloxidase (ProPO) response of caterpillars plotted in nutrient space. $e$, Total hemolymph protein response of caterpillars plotted in nutrient space. $f$, Hemocyte density of caterpillars plotted in nutrient space. 


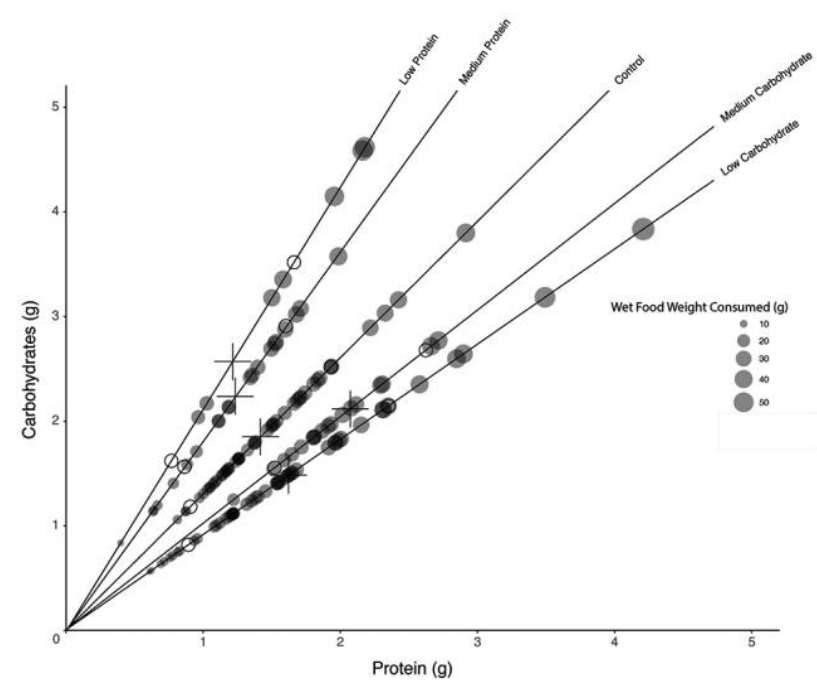

Figure 3. Caterpillar intake plotted in nutrient space. Gray circles represent individual caterpillar intake, large black crosses represent average for each diet treatments, and open circles represent the standard deviation for each diet treatment. Total wet weight of food consumed is mapped to the point size. The $X$ - and $Y$-axes depict the amount of protein and carbohydrates consumed by larvae, respectively. Food intake for reduced-carbohydrate diets, the control diet, and the medium-protein diet were at similar levels, but average intake for the low-protein diet was higher $(t=3.003, P=0.003)$, indicating the presence of compensatory feeding behavior.

different evolutionary histories. Here, we demonstrate the importance of nutritional context in shaping the interaction between performance and immune function. Within individual diets, there was no correlation between performance and immune function (fig. 4), but across all diets, there was a positive correlation (albeit with significant variation around the best-fit models; table 3), indicating that nutrition plays a bigger role in shaping overall levels of performance and immune function than does the internal biological relationship between the two. The absence of trade-offs between different components of organismal function has been demonstrated in many species and discussed extensively (Reznick et al. 2000). Furthermore, the positive correlations we show between some components of immune function and performance are reminiscent of scenarios in which organisms appear not to be subject to trade-offs among different developmental and physiological systems ("superfleas"; Reznick et al. 2000). Our findings match predictions made by researchers about the conditions needed to produce superfleas - namely, that environmental variability (here, nutrient quality) can play a key role.

Though the interaction between nutrition and immune function appears to be complex in our system, a few general patterns arise. First, we find somewhat mixed support for the qualitative resource hypothesis. Overall, protein appears to be more important than carbohydrates in affecting both immune function and performance (fig. 2). In general, decreases in protein resulted in less optimal levels of immune function or performance. Protein plays an important role in insect growth and development (Woods 1999; Ojeda-Avila et al. 2003) and immune function (Lee et al. 2008; Brunner et al. 2014). Protein is also a limiting resource for many insect herbivores, as plant material is particularly low in usable nitrogen (Felton 1996). Though reduction in protein plays an important role in shaping both performance and immune function in our system, the surfaces presented (fig. 2) are still complex, vary across nutrient space, and do not match patterns of energy or single-resource limitation (Cotter et al. 2011). While some of these patterns could be partially explained by the fact that our experimental diets differed slightly in macronutrient density, compared to the control diet, or because developmental stages within the fifth instar were not perfectly synced across treatments, we argue that the majority of surface variation is likely due to changes in the $\mathrm{P}: \mathrm{C}$ ratio and its direct physiological effects. Other experiments examining the performance effects of different macronutrient ratios and densities on developing insects have demonstrated mixed results - animals fed high macronutrient densities (68\%) show reduced growth rates, and the differences in growth rate, pupal mass, and development time often varied more between diets of different $\mathrm{P}: \mathrm{C}$ ratios than across diets with different macronutrient densities (Deans et al. 2015). In addition, the macronutrient reductions used by researchers in this study (up to a $47 \%$ decrease) represent values much higher than the maximum reduction in our study (18\%). In summary, in our study, protein reduction has a bigger impact on growth performance, survivability, and some immune components than carbohydrate reduction, but it can interact with carbohydrate levels in complex ways to shape immune function or performance.

The second pattern that emerges from our results is that the peaks of each of these factors (e.g., highest growth rates and survivorship, greatest encapsulation volume, highest PO levels) are not aligned in nutrient space (fig. 2), suggesting that a single diet composition does not generate optimal function across all physiological systems: different physiological functions require different combinations of nutrients. Overall, these results align with work done in another species of Lepidoptera, Spodoptera littoralis (Cotter et al. 2011). However, specific patterns of immune function and performance differ between the two

Table 2: Effects of experimental diet on Manduca sexta immune function

\begin{tabular}{lccc}
\hline Response & df & $F$ & $P$ \\
\hline Overall & $\mathbf{4}$ & $\mathbf{2 . 3 2 0 8}$ & $\mathbf{. 0 0 2}$ \\
Encapsulation & 4 & 2.2831 & .073 \\
Phenoloxidase & $\mathbf{4}$ & $\mathbf{4 . 8 5 8 5}$ & $\mathbf{. 0 0 2}$ \\
Prophenoloxidase & 4 & 1.1801 & .331 \\
Protein & $\mathbf{4}$ & $\mathbf{5 . 9 0 0 4}$ & $<.001$ \\
Hemocyte density & 4 & 1.5229 & .2098 \\
\hline
\end{tabular}

Note. Data are based on an overall MANOVA model and ANOVAs comparing the effects of diet treatment on individual measures of immune function. Significant values are shown in boldface. 


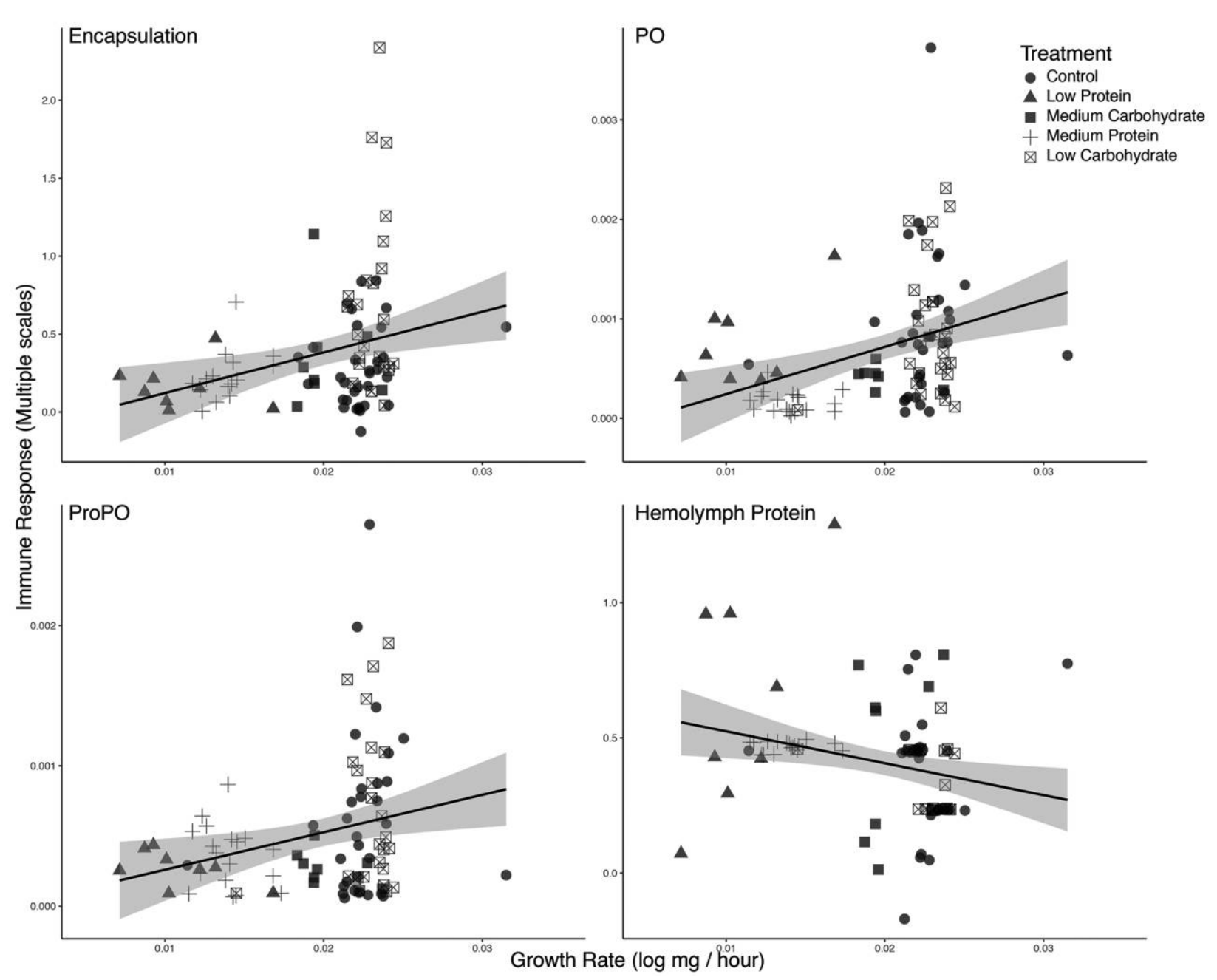

Figure 4. Relationships between Manduca sexta larval performance and four components of the immune system: encapsulation, phenoloxidase (PO), prophenoloxidase (ProPO), and total hemolymph protein. Each symbol represents an individual caterpillar's growth rate (the slope of the individual models plotted in fig. 1) and each component of immune function. Black lines and shaded areas represent the best-fit ordinary least squares regression and $95 \%$ confidence interval for each panel, which model each immune component as a function of growth rate, without regard to diet treatment. PO, ProPO, and encapsulation show significant positive correlations with performance, while total hemolymph protein shows a significant negative correlation. However, this effect is largely due to differences among diet treatments. Hemocyte count showed no correlation with performance (table 3).

species, probably because of either the different dietary requirements of each species (M. sexta is a specialist herbivore, whereas $S$. littoralis is a generalist), the different evolutionary histories and exposure to different pathogens and parasitoids, or the fact that larvae in our study spent their entire developmental period on experimental diets. There are relatively few studies that have examined immune function in the context of nutritional ecology, and these differences between two species raise the question of the generality of patterns of physiological function in nutrient space-are there some systems (or components of systems) that are more limited by single resources than others, or are some systems energy limited? We advocate further examinations of the effects of nutrition on physiological function in other insect systems to begin to answer these questions.
Diet quality in early developmental stages can have longlasting impacts on physiological systems later in development, likely leading to marked changes in growth (Woods 1999) and

Table 3: Effects of growth rate on components of immune function

\begin{tabular}{lcccc}
\hline Immune component & df & $F$ & $P$ & $R^{2}$ \\
\hline Encapsulation & $\mathbf{8 8}$ & $\mathbf{8 . 6 3}$ & $\mathbf{. 0 0 4 2}$ & $\mathbf{. 0 7 9}$ \\
Phenoloxidase & $\mathbf{9 2}$ & $\mathbf{1 3 . 3 9}$ & $\mathbf{. 0 0 0 4}$ & $\mathbf{. 1 1 8}$ \\
Prophenoloxidase & $\mathbf{9 1}$ & $\mathbf{6 . 7 2 2}$ & $\mathbf{. 0 1 1 1}$ & $\mathbf{. 0 5 9}$ \\
Protein & $\mathbf{9 0}$ & $\mathbf{6 . 6 2 8}$ & $\mathbf{. 0 1 1 7}$ & $\mathbf{. 0 5 8}$ \\
Hemocyte density & 69 & .7343 & .3945 & -.003 \\
\hline
\end{tabular}

Note. Significant effects are in boldface. 
immune function. Though some herbivorous insects have the ability to choose between multiple host plants throughout development, many do not-they are often at the mercy of the host plant that they are oviposited on. Manduca sexta is one such insect, and the work here mirrors the experience of a caterpillar in the field - they typically spend their life on a single individual (and single species of) host plant (Mira and Bernays 2002). By measuring performance throughout larval growth and immune function at the end of development, our study mimics an ecologically relevant effect of nutrition on these systems. Manduca sexta is considered a specialist herbivore, feeding exclusively on plants within the family Solanaceae (with one exception, Probiscidea spp. in Martyniaceae). Our work is the first to examine the interplay between nutrition, performance, and immune function with the GF in a specialist insect herbivore. There has been long-standing debate surrounding strategies of specialization in insect herbivores (Bernays and Graham 1988; van der Meijden 1996; Ali and Agrawal 2012), but overall, it is predicted that the range of suitable nutritional space should be smaller for specialists than for generalists, given that they are restricted to a small set of host species (Raubenheimer and Simpson 1999). Here, we show that M. sexta has a relatively narrow window for peak performance (good at all things - different parts of immune function and growth and survival) and that this window is narrowed further when components of immune function are taken into account. Future work could build on the foundation provided here by examining differences in the nutritional quality of different populations or species of host plants or by making comparisons of the nutrient spaces available to different species along the specialistgeneralist gradient. Furthermore, because our experiments were performed with laboratory lines of M. sexta, it would be interesting to compare the effects of nutrition on wild and laboratory lines, as others have done in different contexts (Diamond et al. 2010).

In conclusion, we use the GF to show that the specialist herbivorous caterpillar $M$. sexta shows no trade-off between growth performance and immune function throughout larval development and that diet quality, particularly reductions in protein, has large impacts on both performance and immune function. We demonstrate some evidence for the qualitative resource constraints hypothesis, which indicates that a single diet composition is not optimal across all physiological systems. By better understanding the simultaneous and interactive effects of multiple nutrients on intertwined physiological systems, we gain a greater understanding of how selection acts on the organisms that host these systems and the ecological ramifications of their interaction.

\section{Acknowledgments}

This study was supported in part by National Science Foundation grant IOS- 1053318 to G.D. and by the Center for Insect Science National Institutes of Health Postdoctoral Excellence in Research and Teaching (PERT) grant K12GM000708. We also thank Jose Gutierrez and Meghan Cole for help with logistics and caterpillar measurements.

\section{APPENDIX}

Table A1: Parameters for the random-slopes-and-intercepts linear mixed-effects model used to model log caterpillar weight as a function of time and diet treatment

\begin{tabular}{|c|c|c|c|c|c|c|c|}
\hline Parameter & Estimate & SE & df & $t$ value & $P$ & Correlation & SD \\
\hline \multicolumn{8}{|l|}{ Fixed: } \\
\hline Intercept & 1.1534 & .0754 & 673 & 15.290 & $<.0001$ & & \\
\hline Time & .0221 & .0004 & 673 & 54.226 & $<.0001$ & & \\
\hline Low protein (LP) & .1024 & .1670 & 122 & .613 & .5407 & & \\
\hline Low carbohydrate (LC) & -.0202 & .1073 & 122 & -.188 & .8509 & & \\
\hline Medium protein (MP) & .2557 & .1236 & 122 & 2.069 & .0407 & & \\
\hline Medium carbohydrate (MC) & -.2645 & .1367 & 122 & -1.936 & .0552 & & \\
\hline Time $\times$ LP & -.0114 & .0084 & 673 & -13.488 & $<.0001$ & & \\
\hline Time $\times$ LC & .0004 & .0006 & 673 & .734 & .4633 & & \\
\hline Time $\times$ MP & -.0083 & .0006 & 673 & -12.918 & $<.0001$ & & \\
\hline Time $\times \mathrm{MC}$ & -.0008 & .0007 & 673 & -1.097 & .2731 & & \\
\hline \multicolumn{8}{|l|}{ Random: } \\
\hline Intercept & & & & & & $\ldots$ & .2497 \\
\hline Time & & & & & & -.242 & .0019 \\
\hline Residual & & & & & & $\ldots$ & .5532 \\
\hline
\end{tabular}




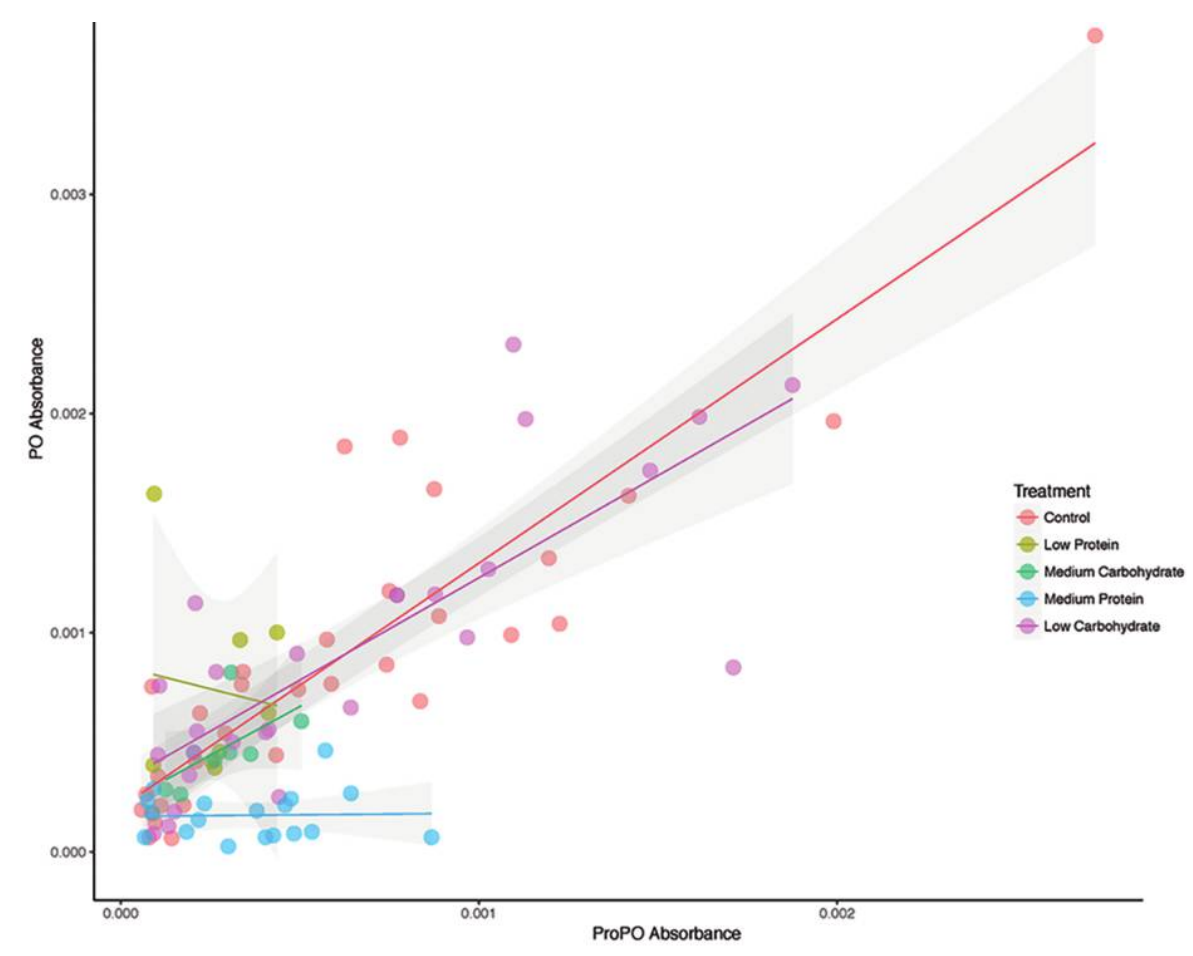

Figure A1. Correlation between prophenoloxidase (ProPO) and phenoloxidase (PO) absorbance values. Points of different colors represent individual caterpillars within each dietary treatment group. Lines represent best-fit linear regression models for each group, with gray bands representing $95 \%$ confidence intervals. While overall trends across all treatment groups demonstrate a strong positive relationship between ProPO and $\mathrm{PO}$, there is substantial variation in this relationship among groups.

\section{Literature Cited}

Ali J.G. and A.A. Agrawal. 2012. Specialist versus generalist insect herbivores and plant defense. Trends Plant Sci 17: 293-302. doi:10.1016/j.tplants.2012.02.006.

Ardia D.R. 2005. Individual quality mediates trade-offs between reproductive effort and immune function in tree swallows. LAnim Ecol 74:517-524. doi:10.1111/j.1365-2656 .2005.00950.x.

Arnold S.J. 1983. Morphology, performance and fitness. Am Zool 23:347-361.

Awmack C.S. and S.R. Leather. 2002. Host plant quality and fecundity in herbivorous insects. Annu Rev Entomol 47: 817-844. doi:10.1146/annurev.ento.47.091201.145300.

Bascuñán-García A.P., C. Lara, and A. Córdoba-Aguilar. 2010. Immune investment impairs growth, female reproduction and survival in the house cricket, Acheta domesticus. IInsect Phvsiol 56:204-211. doi:10.1016/j.jinsphys.2009.10.005.

Bernays E. and M. Graham. 1988. On the evolution of host specificity in phytophagous arthropods. Ecology 69:886-892.

Bonsall M.B. and M.P. Hassell. 1997. Apparent competition structures ecological assemblages. Nature 388:371-373. doi: $10.1038 / 41084$.

Brunner F.S., P. Schmid-Hempel, and S.M. Barribeau. 2014. Protein-poor diet reduces host-specific immune gene ex- pression in Bombus terrestris. Proc R Soc B 281:20140128. doi:10.1098/rspb.2014.0128.

Calder P.C. 2013. Feeding the immune system. Proc Nutr Soc 72:299-309.

Cotter S.C., M. Beveridge, and L.W. Simmons. 2008. Male morph predicts investment in larval immune function in the dung beetle, Onthophagus taurus. Behav Ecol 19:331337. doi:10.1093/beheco/arm137.

Cotter S.C., L.E.B. Kruuk, and K. Wilson. 2004. Costs of resistance: genetic correlations and potential trade-offs in an insect immune system. LEvol Biol 17:421-429. doi:10.1046 /j.1420-9101.2003.00655.x.

Cotter S.C., S.J. Simpson, D. Raubenheimer, and K. Wilson. 2011. Macronutrient balance mediates trade-offs between immune function and life history traits. Funct Ecol 25:186198. doi:10.1111/j.1365-2435.2010.01766.x.

Couture J.J., J.S. Servi, and R.L. Lindroth. 2010. Increased nitrogen availability influences predator-prey interactions by altering host-plant quality. Chemoecology 20:277-284. doi:10.1007/s00049-010-0058-y.

Cressie N. 1990. The origins of kriging. Math Geol 22:239252. doi:10.1007/BF00889887.

Davidowitz G., L.J. D’Amico, and H.F. Nijhout. 2003. Critical weight in the development of insect body size. Evol Dev 5: 188-197. doi:10.1046/j.1525-142X.2003.03026.x. 
Davidowitz G., D. Roff, and H.F. Nijhout. 2016. Synergism and antagonism of proximate mechanisms enable and constrain the response to simultaneous selection on body size and development time: an empirical test using experimental evolution. Am Nat 188:499-520. doi:10.1086/688653.

Deans C.A., S.T. Behmer, J. Fiene, and G.A. Sword. 2016. Spatiotemporal, genotypic, and environmental effects on plant soluble protein and digestible carbohydrate content: implications for insect herbivores with cotton as an exemplar. LChem Ecol 42:1151-1163. doi:10.1007/s10886-016-0772-1.

Deans C.A., G.A. Sword, and S.T. Behmer. 2015. Revisiting macronutrient regulation in the polyphagous herbivore Helicoverpa zea (Lepidoptera: Noctuidae): new insights via nutritional geometry. I Insect Physiol 81:21-27. doi:10 $.1016 /$ j.jinsphys.2015.06.015.

Diamond S.E., S.D. Hawkins, H.F. Nijhout, and J.G. Kingsolver. 2010. Evolutionary divergence of field and laboratory populations of Manduca sexta in response to host-plant quality. Ecol Entomol 35:166-174. doi:10.1111/j.1365-2311.2009 .01166.x.

Dicke M. 2000. Chemical ecology of host-plant selection by herbivorous arthropods: a multitrophic perspective. Biochem Svst Ecol 28:601-617. doi:10.1016/S0305-1978(99)00106-4.

Dominick O.S. and J.W. Truman. 1984. The physiology of wandering behaviour in Manduca sexta. I. Temporal organization and the influence of the internal and external environments. LExp Biol 110:35-51.

Felton GW. 1996. Nutritive quality of plant protein: sources of variation and insect herbivore responses. Arch Insect Biochem Physiol 32:107-130. doi:10.1002/(SICI)1520-6327 (1996)32:1<107::AID-ARCH7>3.0.CO;2-X.

González-Santoyo I. and A. Córdoba-Aguilar. 2012. Phenoloxidase: a key component of the insect immune system. Entomol Exp Appl 142:1-16. doi:10.1111/j.1570-7458.2011.01187.x.

Hung S.Y. and D.G. Boucias. 1996. Phenoloxidase activity in hemolymph of naive and Beauveria bassiana-infected Spodoptera exigua larvae. L Invertebr Pathol 67:35-40. doi:10.1006/jipa .1996 .0006 .

Kaplan I., J. Carrillo, M. Garvey, and P.J. Ode. 2016. Indirect plant-parasitoid interactions mediated by changes in herbivore physiology. Curr Opin Insect Sci 14:112-119. doi: 10.1016/j.cois.2016.03.004.

Karl I., K.H. Hoffmann, and K. Fischer. 2010. Food stress sensitivity and flight performance across phosphoglucose isomerase enzyme genotypes in the sooty copper butterfly. Popul Ecol 52:307-315.

Lee K.P., S.J. Simpson, and K. Wilson. 2008. Dietary proteinquality influences melanization and immune function in an insect. Funct Ecol 22:1052-1061. doi:10.1111/j.1365-2435 .2008.01459.x.

Legendre, P. and L.F.J. Legendre. 2012. Numerical ecology. 3rd ed. Elsevier, Amsterdam.

Lin L.A. and A.R. Ives. 2003. The effect of parasitoid host-size preference on host population growth rates: an example of Aphidius colemani and Aphis glycines. Ecol Entomol 28: 542-550. doi:10.1046/j.1365-2311.2003.00536.x.
Lochmiller R.L. and C. Deerenberg. 2016. Trade-offs in evolutionary immunology: just what is the cost of immunity? Oikos 88:87-98.

Martin T.E., S.K. Auer, R.D. Bassar, A.M. Niklison, and P. Lloyd. 2007. Geographic variation in avian incubation periods and parental influences on embryonic temperature. Evolution 61:2558-2569. doi:10.1111/j.1558-5646.2007.00204.x.

Mayntz D. and S. Toft. 2001. Nutrient composition of the prey's diet affects growth and survivorship of a generalist predator. Oecologia 127:207-213. doi:10.1007/s004420000591.

McCullough E.L. and D.J. Emlen. 2013. Evaluating the costs of a sexually selected weapon: big horns at a small price. Anim Behav 86:977-985. doi:10.1016/j.anbehav.2013.08 .017 .

McGraw K.J., P.M. Nolan, and O.L. Crino. 2011. Carotenoids bolster immunity during moult in a wild songbird with sexually selected plumage coloration. Biol_L Linn Soc 102:560572. doi:10.1111/j.1095-8312.2010.01594.x.

Mira A. and E.A. Bernays. 2002. Trade-offs in host use by Manduca sexta: plant characters vs natural enemies. Oikos 97:387-397. doi:10.1034/j.1600-0706.2002.970309.x.

Miranda V.A., P.D. Navarro, G. Davidowitz, J. Bronstein, and S.P. Stock. 2013. Effect of insect host age and diet on the fitness of the entomopathogenic nematode-bacteria mutualism. Symbiosis 61:145-153. doi:10.1007/s13199-013-0266-7.

Ojeda-Avila T., H.A. Woods, and R.A. Raguso. 2003. Effects of dietary variation on growth, composition, and maturation of Manduca sexta (Sphingidae: Lepidoptera). LInsect Physiol 49:293-306. doi:10.1016/S0022-1910(03)00003-9.

Ponton F., K. Wilson, S.C. Cotter, D. Raubenheimer, and S.J. Simpson. 2011. Nutritional immunology: a multi-dimensional approach. PLoS Pathogens 7:e1002223. doi:10.1371/journal.ppat .1002223.

Rantala M.J. and D.A. Roff. 2005. An analysis of trade-offs in immune function, body size and development time in the Mediterranean field cricket, Gryllus bimaculatus. Funct Ecol 19:323-330.

Rapkin N., K. Jensen, C.R. Archer, C.M. House, S.K. Sakaluk, E. del Castillo, and J. Hunt. 2018. The geometry of nutrient spacebased life-history trade-offs: sex-specific effects of macronutrient intake on the trade-off between encapsulation ability and reproductive effort in decorated crickets. Am Nat 191: $452-474$.

Rattan R.S. 2010. Mechanism of action of insecticidal secondary metabolites of plant origin. Crop Prot 29:913-920. doi:10.1016/j.cropro.2010.05.008.

Raubenheimer D. and S.J. Simpson. 1993. The geometry of compensatory feeding in the locust. Anim Behav 45:953964. doi:10.1006/anbe.1993.1114.

. 1999. Integrating nutrition: a geometrical approach. Entomol Exp Appl 91:67-82. doi:10.1046/j.1570-7458.1999 .00467.x.

Raubenheimer D., S.J. Simpson, and D. Mayntz. 2009. Nutrition, ecology and nutritional ecology: toward an integrated framework. Funct Ecol 23:4-16. doi:10.1111/j.1365 $-2435.2009 .01522 . x$. 
Reznick D., L. Nunney, and A. Tessier. 2000. Big houses, big cars, superfleas and the costs of reproduction. Trends Ecol Evol 15:421-425. doi:10.1016/S0169-5347(00)01941-8.

Rosenthal G.A. and M.R. Berenbaum. 2012. Herbivores: their interactions with secondary plant metabolites. Vol. 2. Ecological and evolutionary processes. 2nd ed. Academic Press, San Diego, CA.

Schmid-Hempel P. 2003. Variation in immune defence as a question of evolutionary ecology. Proc R Soc B 270:357366. doi:10.1098/rspb.2002.2265.

. 2005. Evolutionary ecology of insect immune defenses. Annu Rev Entomol 50:529-551. doi:10.1146/annurev.ento .50.071803.130420.

Schwenke R.A., B.P. Lazzaro, and M.F. Wolfner. 2016. Reproduction-immunity trade-offs in insects. Annu Rev Entomol 61:239-256. doi:10.1146/annurev-ento-010715-023924.

Simpson S.J. and D. Raubenheimer. 2001. The geometric analysis of nutrient-allelochemical interactions: a case study using locusts. Ecology 82:422-439. doi:10.2307/2679870.

Simpson S.J., R.M. Sibly, K.P. Lee, S.T. Behmer, and D. Raubenheimer. 2004. Optimal foraging when regulating intake of multiple nutrients. Anim Behav 68:1299-1311. doi:10 .1016/j.anbehav.2004.03.003.

Soler J.J., L. de Neve, T. Pérez-Contreras, M. Soler, and G. Sorci. 2003. Trade-off between immunocompetence and growth in magpies: an experimental study. Proc R Soc B 270:241-248. doi:10.1098/rspb.2002.2217.

Stearns S.C. 1992. The evolution of life histories. Oxford University Press, Oxford.

Sterner R.W. and J.J. Elser. 2002. Ecological stoichiometry: the biology of elements from molecules to the biosphere. Princeton University Press, Princeton, NJ.

Stiling P. and T. Cornelissen. 2007. How does elevated carbon dioxide $\left(\mathrm{CO}_{2}\right)$ affect plant-herbivore interactions? a field experiment and meta-analysis of $\mathrm{CO}_{2}$-mediated changes on plant chemistry and herbivore performance. Glob Change Biol 13:1823-1842. doi:10.1111/j.1365-2486.2007.01392.x.

Thompson S.N. and R.A. Redak. 2005. Feeding behaviour and nutrient selection in an insect Manduca sexta L. and alterations induced by parasitism. I Comp Physiol A 191:909-923.

van der Meijden E. 1996. Plant defence, an evolutionary dilemma: contrasting effects of (specialist and generalist) herbivores and natural enemies. Entomol Exp Appl 80:307-310. doi:10.1111/j.1570-7458.1996.tb00941.x.

van der Most P.J., B. de Jong, H.K. Parmentier, and S. Verhulst. 2011. Trade-off between growth and immune function: a meta-analysis of selection experiments. Funct Ecol 25:74-80. doi:10.1111/j.1365-2435.2010.01800.x.

Vijendravarma R.K., A.R. Kraaijeveld, and H.C.J. Godfray. 2009. Experimental evolution shows Drosophila melanogaster resistance to a microsporidian pathogen has fitness costs. Evolution 63:104-114. doi:10.1111/j.1558-5646.2008.00516.x.

West G.B., J.H. Brown, and B.J. Enquist. 1999. The fourth dimension of life: fractal geometry and allometric scaling of organisms. Science 284:1677-1679. doi:10.1126/science.284 .5420 .1677 .

Wilson J.K. and H.A. Woods. 2015. Protection via parasitism: Datura odors attract parasitoid flies, which inhibit Manduca larvae from feeding and growing but may not help plants. Oecologia 179:1159-1171. doi:10.1007/s00442-015-3419-y. - 2016. Innate and learned olfactory responses in a wild population of the egg parasitoid Trichogramma (Hymenoptera: Trichogrammatidae). LInsect Sci 16:110. doi:10.1093 /jisesa/iew108.

Woods H.A. 1999. Patterns and mechanisms of growth of fifth-instar Manduca sexta caterpillars following exposure to low-or high-protein food during early instars. Physiol Biochem Zool 72:445-454. 